\title{
The Role of Device Diagnostic Algorithms in the Assessment and Management of Patients with Systolic Heart Failure: A Review
}

\author{
Andrew C. T. Ha, Richard A. Leather, Paul G. Novak, Laurence D. Sterns, \\ and Anthony S. L. Tang
}

Division of Cardiology, Royal Jubilee Hospital, 1952 Bay Street, Victoria, Canada V8R 1J8

Correspondence should be addressed to Anthony S. L. Tang, atang@westerncardiology.ca

Received 12 December 2010; Accepted 3 February 2011

Academic Editor: Gregory Giamouzis

Copyright (c) 2011 Andrew C. T. Ha et al. This is an open access article distributed under the Creative Commons Attribution License, which permits unrestricted use, distribution, and reproduction in any medium, provided the original work is properly cited.

\begin{abstract}
Hospitalization due to heart failure (HF) exacerbation represents a major burden in health care and portends a poor long-term prognosis for patients. As a result, there is considerable interest to develop novel tools and strategies to better detect onset of volume overload, as HF hospitalizations may be reduced if appropriate interventions can be promptly delivered. One such innovation is the use of device-based diagnostic parameters in HF patients with implantable cardioverter defibrillators (ICD) and/or cardiac resynchronization therapy (CRT) devices. These diagnostic algorithms can effectively monitor and detect changes in patients' HF status, as well as predict one's risk of HF hospitalization. This paper will review the role of these device diagnostics parameters in the assessment and management of HF patients in ambulatory settings. In addition, the integration of these novel algorithms in existing HF disease management models will be discussed.
\end{abstract}

\section{Introduction}

Hospitalization for heart failure (HF) is a major health burden upon society, with an estimated prevalence of 5.8 million and an annual incidence of 55000 in the United States alone [1]. In the United States, exacerbation of HF resulted in over 658000 emergence room visits annually, representing a significant economic cost. In addition, hospitalization due to HF portends a poor prognosis for patients, with an estimated in-hospital mortality of 4\% [2]. A major key in reducing HF hospitalization is the early recognition of HF exacerbation, which in turn may lead to prompt diagnosis and management of HF without the need for hospital visits. Unfortunately, bedside clinical parameters are often inaccurate in assessing the true volume status of HF patients [3]. Moreover, symptoms leading to HF hospitalization generally occur later in the decompensation course. For example, dyspnea due to pulmonary congestion was only reported within an average of 3 days prior to hospitalization [4]. Early recognition and prompt management of HF exacerbation has been shown to reduce hospitalization and improve quality of life for HF patients [5,6]. As a result, there is considerable interest to develop strategies that facilitate prompt recognition and management of HF exacerbations in these patients. One such innovation is the use of devicebased diagnostic parameters to assess for signs of volume overload and to predict the onset of HF exacerbation. These functions are integrated within the platforms of certain implantable cardioverter defibrillator (ICD) and cardiac resynchronization therapy (CRT) devices. They are readily accessible from routine device interrogation and are user friendly. The ever expanding indications of ICD and CRT therapy will conceivably make these device-based diagnostic parameters an indispensable tool in HF care. As such, this paper will review the current state of knowledge regarding the use of these device-based diagnostics in the assessment and management of patients with systolic HF.

\section{Heart Rate Variability (HRV)}

Heart rate variability arises from the interplay between the two limbs of the autonomic nervous system in order to 
maintain adequate cardiac output for the body's needs. The parasympathetic input to the heart is primarily responsible for HRV [7]. In humans, chronic heart failure is known to exert deleterious alterations upon cardiac autonomic control [8]. Derangement in the homeostatic balance of the cardiac autonomic system is associated with poorer outcomes and increased mortality $[9,10]$. Among patients with implanted pacing devices, continuous monitoring of HRV could be performed by measurement of the sinus rate from the atrial lead. In these devices, assessment of HRV was achieved by examining the standard deviation of the 5-minute median atrial-to-atrial depolarization interval (SDAAM). Adamson et al. first described the use of HRV as a device-based diagnostic tool in HF patients [11]. In 397 patients with systolic HF and New York Heart Association (NYHA) III or IV symptoms with an implanted CRT, measurements of SDAAM were correlated to their clinical course over an 18month period. An SDAAM < 50 ms (averaged over 4 weeks) was associated with increased all-cause and cardiovascular mortality, with hazard ratios of 3.20 and 4.43, respectively. In addition, SDAAM were persistently depressed during the entire followup among patients who were hospitalized or died. An SDAAM > $100 \mathrm{~ms}$ was associated with a low risk for hospitalization. Moreover, the decline in SDAAM was found to precede HF hospitalization by a median of 16 days and returned to baseline after treatment. If changes in the patient's neurohormonal status precede development of HF symptoms, continuous monitoring of SDAAM may be a valuable tool in predicting one's future risk of HF hospitalization. Indeed, the interrelationship between HRV (as reflected by SDAAM) and HF status has been confirmed by two studies, which showed that a favourable response to CRT therapy was associated with increases in HRV and concomitant decreases of the mean heart rate $[11,12]$. In current devices, measurement of SDAAM depends upon the presence of intrinsic sinus node activity. As such, the clinical use of SDAAM cannot be applied to patients with persistent atrial fibrillation (AF) or if atrial pacing is required $>80 \%$ over a 24 -hour period, since SDAAM cannot be accurately measured in these situations [11]. Taken together, however, measurement of HRV by SDAAM appears to be a valid clinical tool in assessing one's HF status and risk of subsequent HF hospitalization.

\section{Intrathoracic Impedance}

Assessment of thoracic fluid status by measuring intrathoracic impedance is the newest device-based diagnostic tool available in clinical practice. This is based on the concept that changes of fluid volume in the lungs will alter intrathoracic impedance $[13,14]$. The OptiVol fluid status monitoring algorithm (Medtronic, Inc., Minneapolis, USA) is incorporated in contemporary ICD and CRT-D platforms. In these systems, intrathoracic impedance is measured between the pulse generator (usually implanted in the left pectoral region) and the right ventricular coil of the ICD lead. This vector encompasses most of the left thoracic cavity and defines the intrathoracic impedance as measured by the device. Using the concept of Ohm's law, impedance of the hemithorax cavity is measured upon delivery of a small alternating current between the pulse generator and the ICD lead. Since fluid (water, blood) is a highly conductive medium, accumulation of fluid in the lungs will lower intrathoracic impedance. A purported advantage of this algorithm is the reproducibility and consistency of repeated measurements due to the fixed positions of the two electrodes [15]. This makes data trending possible and allows clinicians to follow the pulmonary fluid status of patients over time.

The OptiVol fluid status monitor is activated $\geq 34$ days after the index procedure in order to prevent spurious measurements due to air and edema in the healing pocket. Once active, the device will measure the intrathoracic impedance every 20 minutes between noon and $5 \mathrm{pm}$, totalling 64 recordings. These values are averaged and reported as the "daily impedance value". Daily measurements of impedance are compared to the patient's reference value, which is derived from the average of the last 4 daily impedance recordings. Negative deviations of the daily impedance value generate the OptiVol fluid index (Figure 1). The OptiVol index has a unit measurement of ohms - days and is compared to a programmable fluid index which is nominally set as $60 \mathrm{ohms}$ - days. The OptiVol fluid index is plotted against time to provide two pieces of information: (i) the magnitude of the deviation from the programmable fluid index threshold; (ii) the time duration of the deviation. Pulmonary fluid retention will generate negative deviations of the daily impedance from the reference value, which is graphically depicted as deviations from the zero baseline value in the OptiVol index.

The OptiVol index only provides an indirect measurement of pulmonary congestion based on intrathoracic impedance changes. As such, false-positive and false-negative measurements can occur and need to be borne in mind when this tool is applied in clinical practice. For example, air trapping in emphysematous lungs may mask decreases in intrathoracic impedance. On the other hand, alveolar congestion from pneumonia or the presence of pleural effusions will lower daily impedance measurements even though pulmonary congestion due to elevation of left-sided cardiac pressures had not occurred. Furthermore, not all patients experience pulmonary congestion as their dominant HF exacerbation symptom. Thus, the OptiVol algorithm may not be useful in assessing patients with predominant rightsided heart failure symptoms, or those with low cardiac output despite being relatively euvolemic. In spite of these potential limitations, considerable interests exist in utilizing the OptiVol fluid index to prevent, assess, and manage heart exacerbation events due to pulmonary congestion in patients with implantable cardiac devices.

\section{Clinical Data}

The first proof-of-concept study of OptiVol use in humans was reported by $\mathrm{Yu}$ et al. in the Medtronic Impedance Diagnostics in Heart Failure Patients Trial (Mid-HeFT) [16]. A special pacemaker with an ICD lead was implanted in 33 patients with systolic HF and NYHA class III or IV symptoms. Daily intrathoracic impedance was recorded 


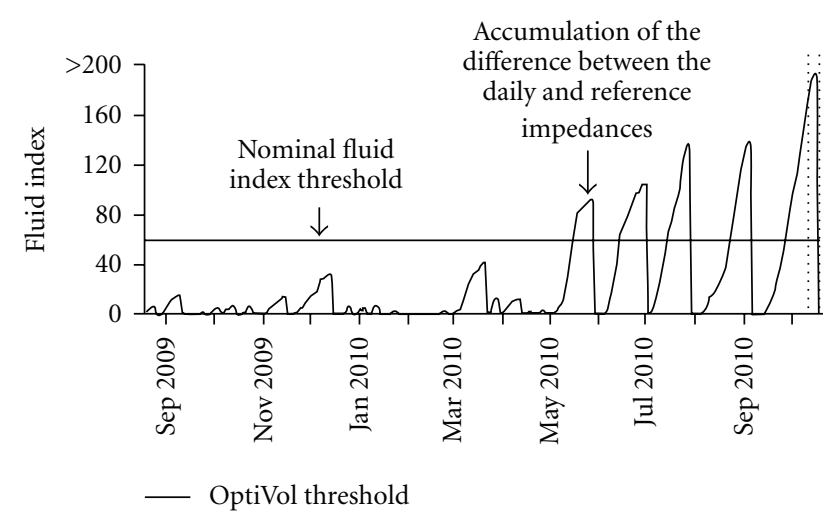

(a)

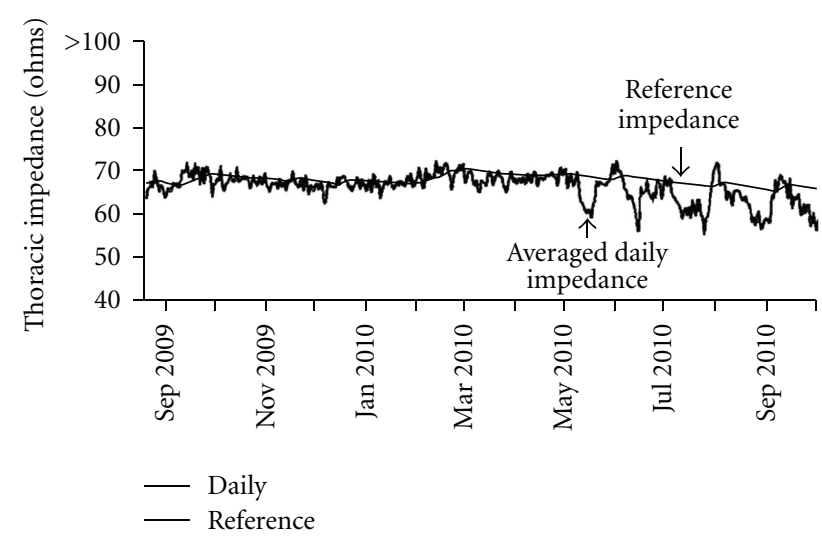

(b)

Figure 1: OptiVol fluid index. The intrathoracic impedance is measured on a daily basis and is compared to the patient's "reference" value, which is derived from the average of the last 4 daily measurements. Negative deviations from the reference value suggest a decrease in intrathoracic impedance, which may reflect pulmonary congestion. The difference between the measured intrathoracic impedance and the reference value is multiplied by time (measured in days) to generate the OptiVol fluid index, which has a unit measurement of ohms · days. The magnitude of the fluid index value is determined by (i) the absolute difference between the reference value and the measured intrathoracic impedance, and/or (ii) the time duration of the difference. The fluid index is plotted against time to graphically display the onset and duration of negative deviations of intrathoracic impedance. The nominal cutoff threshold is set as $60 \mathrm{ohms} \cdot$ days.

by the device, and physicians were blinded to these measurements. In the acute phase of the study, patients with decompensated HF were hospitalized in the coronary care unit and treated with intravenous diuretics and vasodilators. The pulmonary capillary wedge pressures (PCWP) of these patients were measured by pulmonary artery catheters every 2 hours. Intrathoracic impedance was measured by the device every 6 hours in this patient subgroup. A total of 9 patients experienced 24 hospitalizations for HF exacerbation, and PCWP measurements were recorded in 5 patients with 14 hospitalizations. A statistically significant inverse relationship was noted between the PCWP and intrathoracic impedance. As patients were being diuresed, a concomitant increase in intrathoracic impedance was noted. In addition, a statistically significant inverse correlation was demonstrated between net fluid loss during hospitalization and intrathoracic impedance. Furthermore, a decrease in intrathoracic impedance preceded the onset of dyspnea by $15.3 \pm 10.3$ days (mean \pm standard deviation), which was considerably earlier than the actual onset of symptoms which led to hospitalization $(3.0 \pm 2.5$ days $)$. The drop in intrathoracic impedance which occurred before onset of HF symptoms and at HF admission were significantly reduced $(P<.001)$. Thus, the authors conclude that OptiVol may help detect early onset of volume overload, which in turn may predict onset of HF exacerbation.

In the chronic phase of the study, patients were followed by an ambulatory heart function clinic on a regular basis and were managed by physicians who had no knowledge of the intrathoracic impedance measurements. If a patient was hospitalized for HF exacerbation, the intrathoracic impedance values of the preceding 30 days were used to generate the diagnostic parameters of OptiVol. The algorithm development data was derived from 7 patients (11 hospitalizations from 4 patients) and was validated in a cohort of 23 patients (13 hospitalizations from 8 patients). A receiver-operator curve ("performance curve") was constructed to assess the diagnostic sensitivity and specificity of varying thresholds for impedance changes which may predict HF hospitalization. Based on these results, a cutoff value below $60 \Omega$. days was able to detect impedance changes preceding HF hospitalization with a sensitivity of $76.9 \%$. Although the specificity of this cut-off value in predicting HF hospitalization was not explicitly stated in the study, the authors reported a false-positive rate of 1.5 detections per patient-monitoring year. On the basis of this study, the nominal cut-off threshold for pulmonary congestion detection is set as $60 \Omega$. days in the OptiVol algorithm.

The diagnostic performance of the OptiVol algorithm in a "real-world" population was reported by Vollmann et al. from an observational registry of 373 patients implanted with the Medtronic InSync Sentry CRT-D device [18]. In this study, an audible alert was programmed when the intrathoracic impedance fell below the cut-off threshold. Patient followup included regular clinic appointments and unscheduled visits due to alert activation or clinical HF deterioration. A clinical HF exacerbation diagnosed within 2 weeks of the initial alert was classified as a "truepositive alert event". The treating physicians had knowledge of the OptiVol measurements. A total of 53 alert events occurred in 45 patients, and 53 clinical HF exacerbation events occurred in 43 patients. OptiVol was able to detect clinical HF exacerbation with an adjusted sensitivity of $60 \%$ and a positive predictive value of $60 \%$. The falsepositive detection rate was 0.2 event per patient year. In more than half of the clinical HF events (55\%) which did not trigger an alert, the intrathoracic impedance had decreased below the reference value but had not crossed the programmed cut-off threshold. Thus, the authors suggested that the diagnostic sensitivity of OptiVol might be improved if the cut-off threshold values could be individualized. 
TABLE 1: Device diagnostics parameters and algorithms from the Cardiac Compass report.

\begin{tabular}{|c|c|c|}
\hline $\begin{array}{l}\text { Device } \\
\text { diagnostic } \\
\text { parameter }\end{array}$ & Description & Algorithm \\
\hline AF duration & Total amount of time spent during AF on a daily basis. & $\begin{array}{l}\mathrm{AF} \geq 6 \mathrm{~h} \text { on } \geq 1 \text { day in patients without persistent } \mathrm{AF} \text { ( } 7 \\
\text { consecutive days with } \geq 23 \mathrm{~h} \mathrm{AF} \text { ). }\end{array}$ \\
\hline $\begin{array}{l}\text { Ventricular rate } \\
\text { during } \mathrm{AF}\end{array}$ & The daily average ventricular rate during AF. & $\begin{array}{l}\mathrm{AF}=24 \mathrm{~h} \text { and the average ventricular rate } \geq 90 \\
\text { beats/min during } \mathrm{AF} \text { on } \geq 1 \text { day. }\end{array}$ \\
\hline $\begin{array}{l}\text { Fluid index } \\
\text { (OptiVol) }\end{array}$ & $\begin{array}{l}\text { The fluid index trend is the cumulative difference } \\
\text { between the daily average and patient-specific reference } \\
\text { intrathoracic impedance. }\end{array}$ & $\begin{array}{l}\text { High fluid index on } \geq 1 \text { day; thresholds included } \geq 60 \\
\geq 80 ; \geq 100 \Omega \cdot \text { days. }\end{array}$ \\
\hline Patient activity & $\begin{array}{l}\text { Measures the total active time per day using a capacitive } \\
\text { accelerometer. A minute is considered active if the } \\
\text { count exceeds a threshold equivalent to walking } 70 \\
\text { steps/min. }\end{array}$ & Average patient activity $<1 \mathrm{~h}$ over 1 week. \\
\hline Night heart rate & Measures the ventricular rate from midnight to $4 \mathrm{AM}$. & $\begin{array}{l}\text { Average night heart rate }>85 \text { beats } / \text { min for } 7 \\
\text { consecutive days. }\end{array}$ \\
\hline $\begin{array}{l}\text { Heart rate } \\
\text { variability } \\
(\mathrm{HRV})\end{array}$ & $\begin{array}{l}\text { HRV is assessed by the SDAAM (standard deviation of } \\
\text { the } 5 \text {-minute median atrial rate). HRV is not measured } \\
\text { if atrial pacing occurs }>80 \% \text { of the time or if the patient } \\
\text { is in AT/AF. }\end{array}$ & $\begin{array}{l}\text { HRV }<60 \text { ms everyday for } 1 \text { week (minimum } 5 \\
\text { consecutive days). }\end{array}$ \\
\hline $\begin{array}{l}\text { Percentage of } \\
\text { CRT pacing }\end{array}$ & Percentage of biventricular pacing on each day. & Biventricular pacing $<90 \%$ for 5 of 7 days. \\
\hline $\begin{array}{l}\text { ICD shocks for } \\
\text { VT or VF }\end{array}$ & $\begin{array}{l}\text { Records if an ICD shock was delivered for episodes } \\
\text { detected within the VT or VF zone; includes both } \\
\text { appropriate and inappropriate shock(s). }\end{array}$ & $\geq 1$ shock(s) during the evaluation period. \\
\hline
\end{tabular}

Adapted from [17].

$\mathrm{AF}=$ atrial fibrillation; $\mathrm{AT} / \mathrm{AF}=$ atrial tachycardia/atrial fibrillation; $\mathrm{CRT}=$ cardiac resynchronization therapy; $\mathrm{HF}=$ heart failure; $\mathrm{HRV}=$ heart rate variability; ICD = implantable cardioverter defibrillator; $\mathrm{VF}=$ ventricular fibrillation; $\mathrm{VT}=$ ventricular tachycardia.

Programmable fluid index thresholds are available in Medtronic ICD and CRT-D platforms.

Although the use of individual HF device diagnostic parameters has been validated in multiple clinical studies, each parameter has potential limitations which may restrict its general applicability in a complex HF population. As a result, there is considerable interest in combining HF device diagnostic parameters in the management of HF patients with implanted devices. The PARTNERS HF (Program to Access and Review Trending Information and Evaluate Correlation to Symptoms in Patients with Heart Failure) study was a multicenter, prospective observational study which evaluated the use of combined HF diagnostic information in predicting clinical deterioration of ambulatory HF patients [17]. A cohort of 694 patients with systolic HF and NYHA III or IV symptoms with Medtronic CRT-D devices were prospectively evaluated in 100 centers in the United States. Individual HF diagnostic parameters were collected from the Cardiac Compass report (Figure 2), which consisted of (i) atrial fibrillation (AF) duration; (ii) ventricular rate during AF; (iii) fluid index (OptiVol); (iv) patient activity; (v) night heart rate; (vi) heart rate variability; (vii) percentage of CRT pacing; (viii) ICD shocks for ventricular arrhythmias (Figure 1). The definition of a "positive" event for each parameter was prespecified (Table 1). Occurrence of HF events were prospectively collected and independently adjudicated. A combined HF device diagnostic algorithm was developed by the authors and was triggered when (i) the fluid index exceeds $100 \Omega$ - days or (ii) any 2 of the 8 prespecified parameters were positive. In total, 90 patients had 141 adjudicated HF hospitalizations with pulmonary congestion. The combined HF device diagnostic algorithm was triggered in 43\% (298 of 694) of patients and in $23 \%$ (1324 of 5693) of device evaluations. Having $\geq 2$ of the 8 criteria being positive accounted for the majority (72\%) of the algorithm triggers. Importantly, patients with a positive combined HF device diagnostic were at greater risk of HF hospitalization with pulmonary congestion in the next month than those without (adjusted hazard ratio: $4.8,95 \%$ confidence interval: 2.9 to $8.1, P<.0001)$. Among patients with a negative combined HF device diagnostic, the risk of HF hospitalization due to pulmonary congestion was $0.7 \%$ over the next 30 days. In subgroup analyses, the predictive ability of the combined HF device diagnostic algorithm was greater when device evaluations were performed monthly and semimonthly when compared to every 3 months. However, the diagnostic utility of this algorithm appeared to be limited to patients without prior HF events. These observational studies highlight the promising role of OptiVol and other device-based diagnostics in the early detection of pulmonary congestion in HF patients. Whether the use of such device diagnostics can improve outcomes in HF patients with ICD or CRT-D is currently examined by two randomized trials, the Diagnostic Trial in Heart Failure (DOT-HF) in Europe and the Prospective, Randomized Evaluation of Cardiac Compass with OptiVol in the Early 
Cardiac compass report

One or more shocks/day

Treated VT/VF

(episodes/day)

V. rate during VT/VF (bpm)

- VF

- VT

Nonsustained VT

(episodes/day)

AT/AF total (minutes/day)

V. rate during AT/AF

(bpm)

! max/day

Pacing/day (\%)

- Atrial

— Ventricular

Avg v. rate (bpm)

— Day

— Night

Patient activity

(hours/day)

OptiVol fluid index

- OptiVol threshold
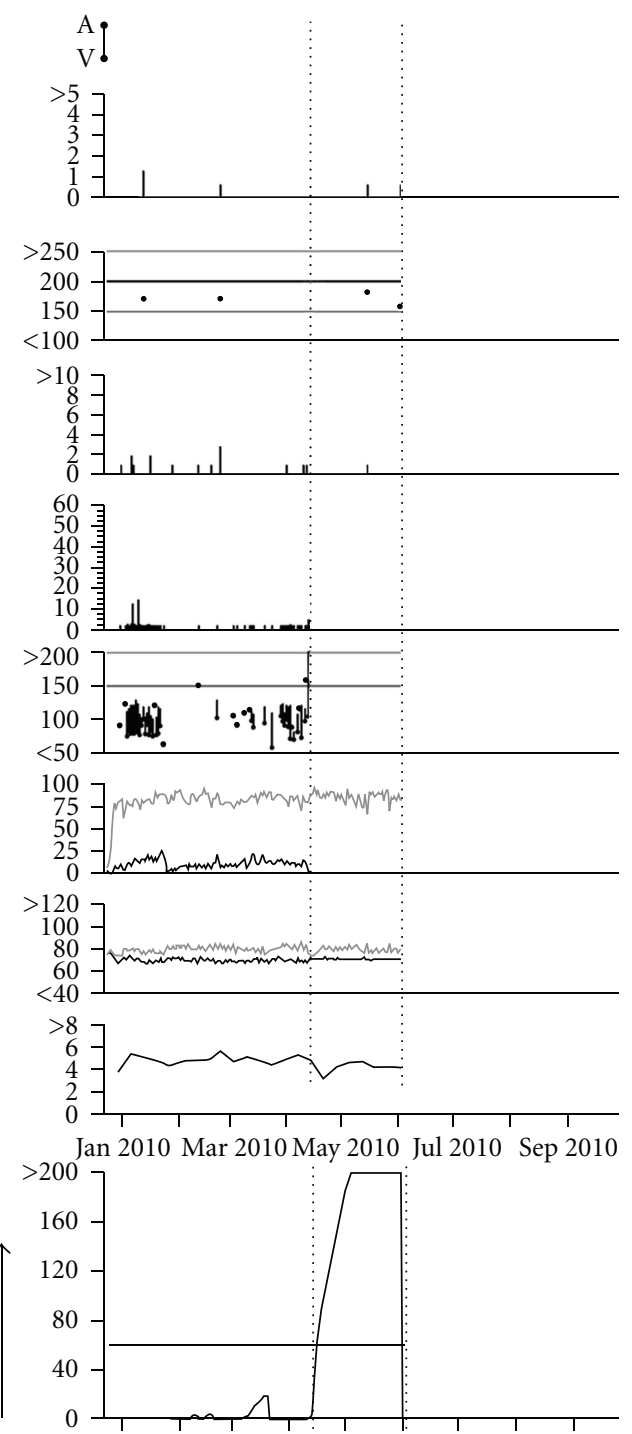

Fluid

Thoracic impedance (ohms)

— Daily
— Reference

Heart rate variability

(ms)

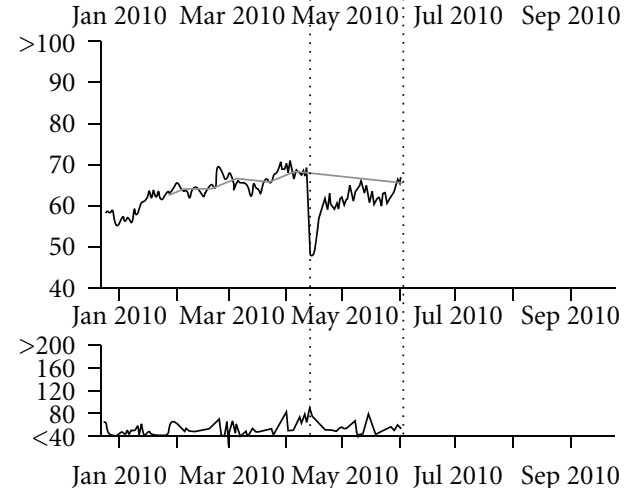

Figure 2: Cardiac Compass report. The Cardiac Compass report displays a number of device diagnostic parameters over a period of weeks to months. This allows trending of data over time, allowing clinicians to monitor the heart failure status of patients. An abnormal reading (such as a fluid index threshold crossing) may reflect a worsening of the patient's heart failure (HF) status, potentially alerting the physician to a possible HF exacerbation in the near future. The combined use of multiple device parameters may improve the diagnostic sensitivity of early HF detection. 
Detection of Decompensation Events for Heart Failure (PRECEDE-HF) trial in North America.

\section{Integration of Device Diagnostics into Heart Failure Clinics}

The feasibility of routine OptiVol assessment in an HF disease management program was recently reported by Mullens et al. [19]. In this pilot study, changes in the fluid index were assessed in $194 \mathrm{HF}$ patients with an implanted ICD or CRT-D via an online remote monitoring system (Carelink, Medtronic, Inc.). Regular Carelink uploads were reviewed by an HF clinic nurse. If the fluid index crossed the nominal threshold ( $60 \Omega$. days) for $\geq 10$ days, a followup telephone call was performed by a nurse. Over a period of 4 months, 400 Carelink uploads were received and 44 fluid index threshold crossings were noted in 34 patients. Thirty-two of the 34 (97\%) patients reported occurrence of "clinically relevant events" at around the time of fluid index threshold crossing, which included HF hospitalization (18\%); changes in HF therapy (56\%); drug and/or dietary nonadherence $(38 \%)$. The incidence of clinically relevant events was not reported in the remaining 160 patients who did not cross the fluid index threshold. In this study, an internet-based program to detect fluid index threshold crossing yielded a high rate of clinically relevant events in a "real-world" population of ambulatory HF patients with implanted pacing devices. The authors concluded that use of OptiVol in an established HF program is feasible and will provide additional useful clinical information to medical providers.

\section{Conclusions}

The expanding implant indications for ICD and CRT will result in increasing numbers of HF patients receiving these life-saving therapies. Clinical studies had demonstrated the utility of certain device-based diagnostic parameters in assessing pulmonary congestion and in predicting one's risk of future HF hospitalization. In particular, intrathoracic impedance assessment by OptiVol is a promising tool in detecting early signs of pulmonary congestion, which in turn may reduce hospitalization with prompt recognition and treatment. The combined use of various device diagnostics has recently been shown to be a powerful predictor of shortterm HF hospitalization risk. Ongoing randomized trials are being performed to evaluate whether these algorithms can improve patient outcomes. If these algorithms can indeed improve HF outcomes, how best to integrate them into existing HF disease management models will certainly become an active area of research.

\section{Acknowledgments}

Dr. R. A. Leather has received speaker honoraria from Medtronic, Inc. and has participated in research studies supported by Medtronic, Inc. Dr. P. G. Novak has received speaker honoraria from Medtronic, Inc., and has participated in research studies supported by Medtronic, Inc. Dr.
L. D. Sterns has received speaker honoraria from Medtronic, Inc. He has participated in research studies supported by Medtronic, Inc. and served as a consultant for Medtronic, Inc. Dr. A. S. L. Tang has received speaker honoraria from Medtronic, Inc. and has participated in research studies supported by Medtronic. Dr. A. C. T. Ha has no conflicts of interest.

\section{References}

[1] N. L. Weintraub, S. P. Collins, P. S. Pang et al., "Acute heart failure syndromes: emergency department presentation, treatment, and disposition: current approaches and future aims: a scientific statement from the American Heart Association," Circulation, vol. 122, no. 19, pp. 1975-1996, 2010.

[2] W. T. Abraham, K. F. Adams, G. C. Fonarow et al., "Inhospital mortality in patients with acute decompensated heart failure requiring intravenous vasoactive medications: an analysis from the Acute Decompensated Heart Failure National Registry (ADHERE)," Journal of the American College of Cardiology, vol. 46, no. 1, pp. 57-64, 2005.

[3] L. W. Stevenson and J. K. Perloff, "The limited reliability of physical signs for estimating hemodynamics in chronic heart failure," Journal of the American Medical Association, vol. 261, no. 6, pp. 884-888, 1989.

[4] S. Chakko, D. Woska, H. Martinez et al., "Clinical, radiographic, and hemodynamic correlations in chronic congestive heart failure: conflicting results may lead to inappropriate care," American Journal of Medicine, vol. 90, no. 3, pp. 353359, 1991.

[5] M. W. Rich, V. Beckham, C. Wittenberg, C. L. Leven, K. E. Freedland, and R. M. Carney, "A multidisciplinary intervention to prevent the readmission of elderly patients with congestive heart failure," The New England Journal of Medicine, vol. 333, no. 18, pp. 1190-1195, 1995.

[6] F. A. McAlister, S. Stewart, S. Ferrua, and J. J. J. V. McMurray, "Multidisciplinary strategies for the management of heart failure patients at high risk for admission: a systematic review of randomized trials," Journal of the American College of Cardiology, vol. 44, no. 4, pp. 810-819, 2004.

[7] H. V. Huikuri, T. Mäkikallio, K. E. J. Airaksinen, R. Mitrani, A. Castellanos, and R. J. Myerburg, "Measurement of heart rate variability: a clinical tool or a research toy?" Journal of the American College of Cardiology, vol. 34, no. 7, pp. 1878-1883, 1999.

[8] A. Mortara, M. T. La Rovere, G. D. Pinna et al., "Arterial baroreflex modulation of heart rate in chronic heart failure: clinical and hemodynamic correlates and prognostic implications," Circulation, vol. 96, no. 10, pp. 3450-3458, 1997.

[9] J. Nolan, P. D. Batin, R. Andrews et al., "Prospective study of heart rate variability and mortality in chronic heart failure: results of the United Kingdom heart failure evaluation and assessment of risk trial (UK-Heart)," Circulation, vol. 98, no. 15, pp. 1510-1516, 1998.

[10] M. T. La Rovere, G. D. Pinna, R. Maestri et al., "Short-term heart rate variability strongly predicts sudden cadiac death in chronic heart failure patients," Circulation, vol. 107, no. 4, pp. 565-570, 2003.

[11] P. B. Adamson, A. L. Smith, W. T. Abraham et al., "Continuous autonomic assessment in patients with symptomatic heart failure: prognostic value of heart rate variability measured by an implanted cardiac resynchronization device," Circulation, vol. 110, no. 16, pp. 2389-2394, 2004. 
[12] C. Fantoni, S. Raffa, F. Regoli et al., "Cardiac resynchronization therapy improves heart rate profile and heart rate variability of patients with moderate to severe heart failure," Journal of the American College of Cardiology, vol. 46, no. 10, pp. 1875-1882, 2005.

[13] R. V. Luepker, J. R. Michael, and J. R. Warbasse, “Transthoracic electrical impedance: quantitative evaluation of a non-invasive measure of thoracic fluid volume," American Heart Journal, vol. 85, no. 1, pp. 83-93, 1973.

[14] L. Wang, S. Lahtinen, L. Lentz et al., "Feasibility of using an implantable system to measure thoracic congestion in an ambulatory chronic heart failure canine model," Pacing and Clinical Electrophysiology, vol. 28, no. 5, pp. 404-411, 2005.

[15] L. Wang, "Fundamentals of intrathoracic impedance monitoring in heart failure," American Journal of Cardiology, vol. 99, no. 10, pp. S3-S10, 2007.

[16] C. M. Yu, L. Wang, E. Chau et al., "Intrathoracic impedance monitoring in patients with heart failure: correlation with fluid status and feasibility of early warning preceding hospitalization," Circulation, vol. 112, no. 6, pp. 841-848, 2005.

[17] D. J. Whellan, K. T. Ousdigian, S. M. Al-Khatib et al., "Combined heart failure device diagnostics identify patients at higher risk of subsequent heart failure hospitalizations. Results from PARTNERS HF (Program to Access and Review Trending Information and Evaluate Correlation to Symptoms in Patients With Heart Failure) Study," Journal of the American College of Cardiology, vol. 55, no. 17, pp. 1803-1810, 2010.

[18] D. Vollmann, H. Nägele, P. Schauerte et al., "Clinical utility of intrathoracic impedance monitoring to alert patients with an implanted device of deteriorating chronic heart failure," European Heart Journal, vol. 28, no. 15, pp. 1835-1840, 2007.

[19] W. Mullens, L. P.J. Oliveira, T. Verga, B. L. Wilkoff, and W. H. Wilson Tang, "Insights from internet-based remote intrathoracic impedance monitoring as part of a heart failure disease management program," Congestive Heart Failure, vol. 16 , no. 4, pp. 159-163, 2010. 


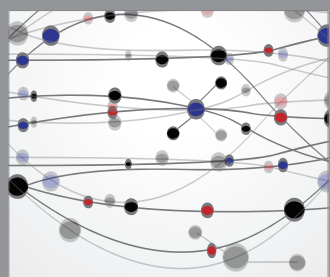

The Scientific World Journal
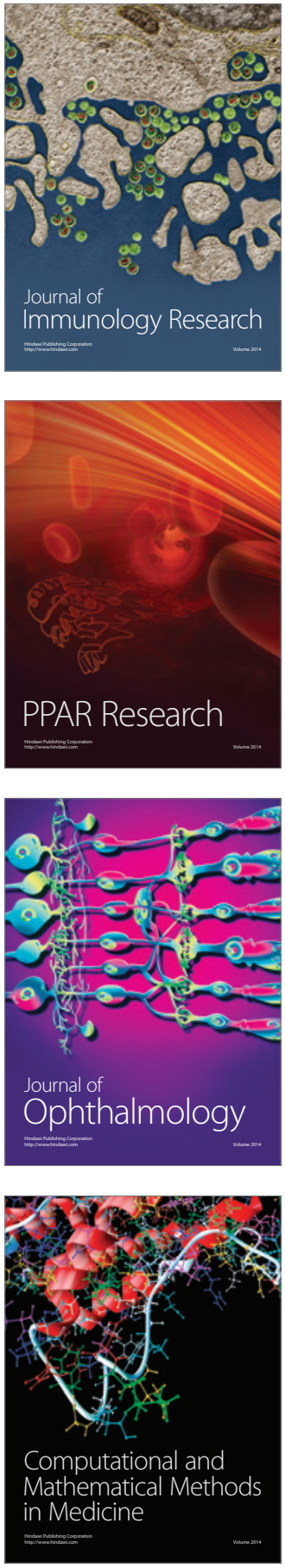

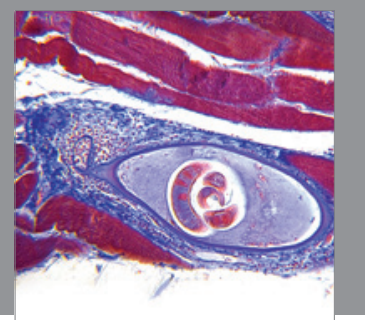

Gastroenterology

Research and Practice
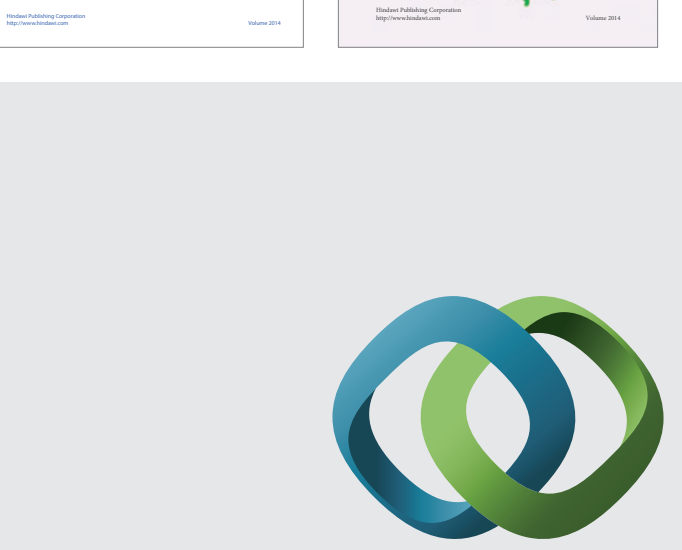

\section{Hindawi}

Submit your manuscripts at

http://www.hindawi.com
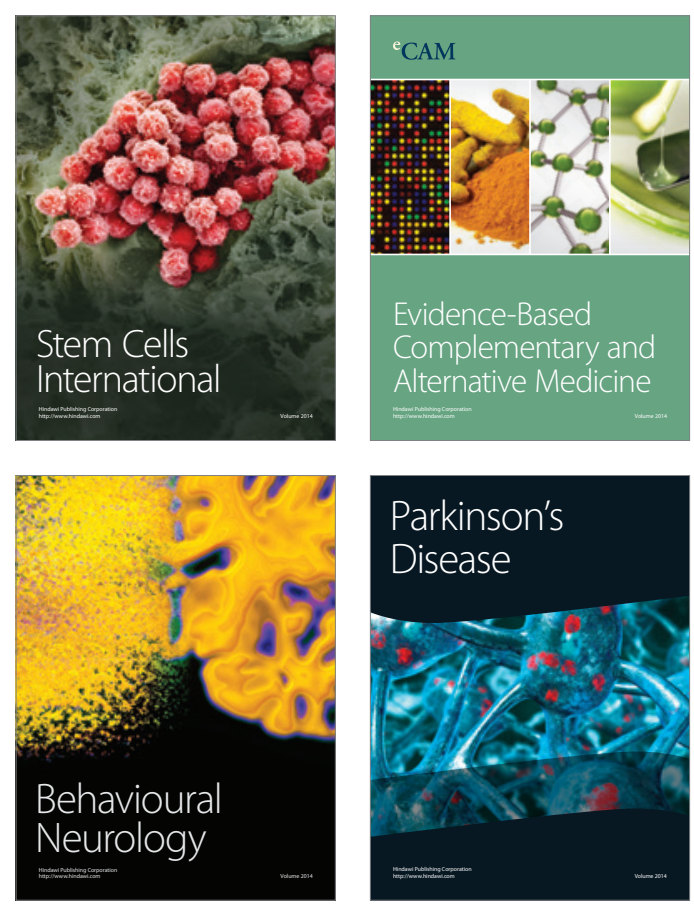

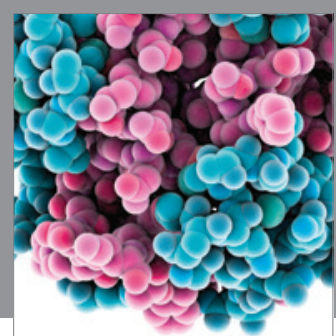

Journal of
Diabetes Research

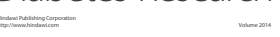

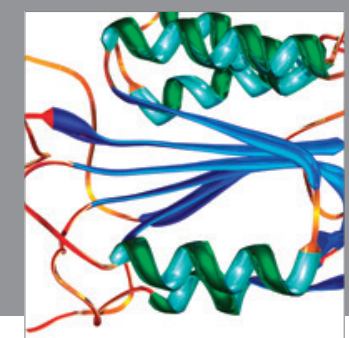

Disease Markers
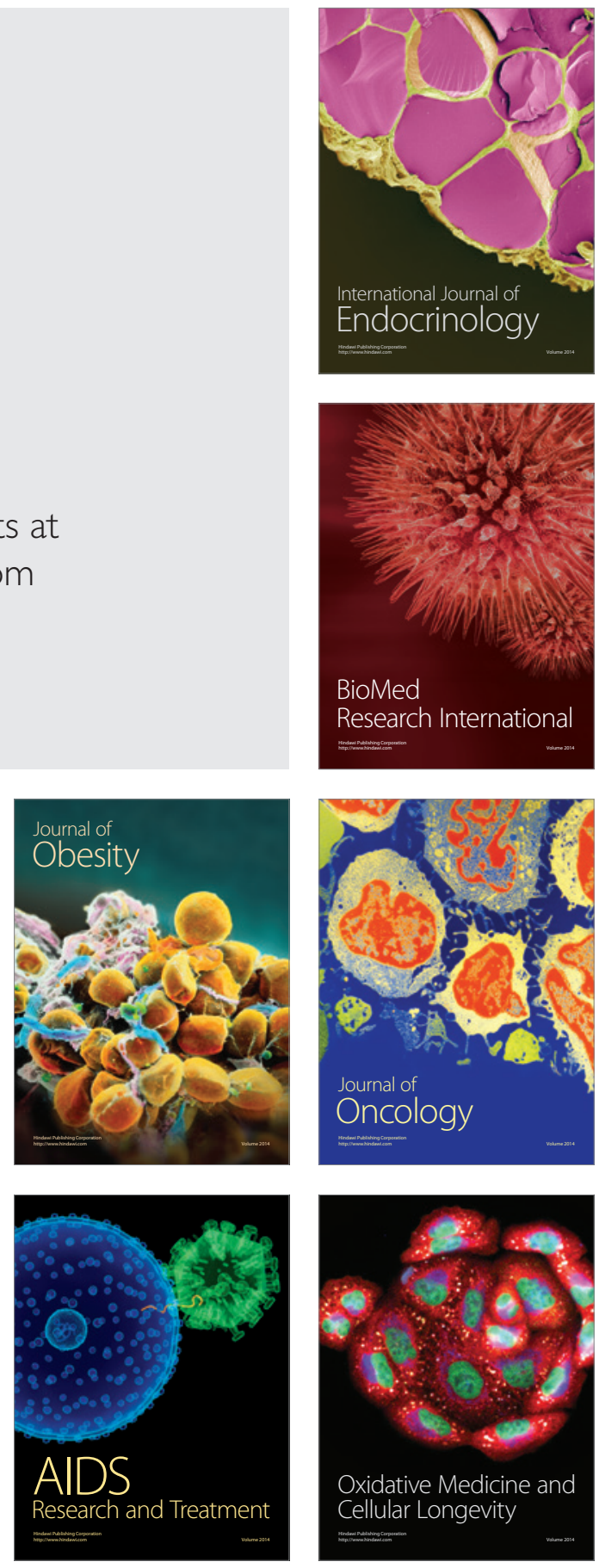